\title{
Methodological Issues in the Study of Prospective Timing
}

\author{
Giovanna Mioni
}

\section{1 \\ Introduction}

The ability to accurately estimate the passage of time plays an important role in daily activities, from the sleep-wake cycle to speaking, to the ability to play musical instruments. We process time across a wide range of intervals, which operates over the range of milliseconds to the 24-h (Buhusi \& Meck, 2005; Fraisse, 1984; Grondin, 2010). As described by Block, Grondin, and Zakay (Chapter 2, this volume), the methods to investigate temporal processing can be distinguished in prospective and retrospective paradigms. In the prospective paradigm, participants know in advance that they will be asked to judge the duration of a time period. In the retrospective paradigm, participants do not know until the end of a time period that they will be asked to judge its duration. The way participants experience the passage of time and the various cognitive processes involved may nevertheless differ between the two paradigms. In the prospective paradigm, a person may intentionally encode temporal information as an integral part of the experience of the time period. In the retrospective paradigm, a person may incidentally encode temporal information, and whatever information is relevant may be later retrieved from memory.

Traditionally, processing duration in the milliseconds-seconds range has been explained using the pacemaker-accumulator model (Gibbon, Church, \& Meck, 1984; Triesman, 1963). The model postulates that duration judgments go through three stages: clock, memory, and decision stage. The clock stage is composed of a pacemaker that emits pulses gated to an accumulator. A switch is placed before the accumulator that controls the flow of pulses from the pacemaker to the accumulator. The memory stage is conceptualized as the storing system that accumulates pulses in working memory for comparison with the content of reference memory. The reference memory contains a long-term memory representation of pulses accumulated across prior trials. The final stage is the decision stage, in which the current duration is compared with those stored in the reference memory. In Treisman's model (1963), 
the pacemaker directly receives inputs from an arousal center. As the arousal levels vary, the speed with which the pacemaker emits pulses change. An increased level of arousal leads to an increase in the speed of the pacemaker. For a given duration period, if the pacemaker runs faster, more pulses reach the accumulator, and this duration is judged to be longer (Droit-Volet \& Meck, 2007). The Attentional-Gate Model (Block \& Zakay, 1996; Zakay \& Block, 1996) acknowledges the role of arousal in the pacemaker speed but it also adds an 'attentional gate' that is influenced by the amount of attention allocated to time. If less attention is devoted to time, the gate narrows and allows fewer pulses to transfer to the accumulator.

These models provide interesting frameworks to test research hypotheses. But how do we test them? Which method is more appropriate for a specific question? In this view, the purpose of this chapter is to facilitate a future researcher that approaches the study of timing to select the appropriate method with a specific focus on prospective timing. Here, the principal methods for studying time in the prospective paradigm will be described. Moreover, applications in clinical populations will be also described.

\section{2 Methods for Studying Time in the Prospective Paradigm}

Traditionally, researchers have utilized many different methods for investigating time (Block, 1990; Grondin, 2008; 2010; Zakay 1990; 1993), however, this chapter, will focus only on the most classical ones used in the prospective paradigm (i.e., time production, verbal estimation, and time reproduction).

Time production and verbal estimation tasks may be considered the two sides of the same coin and reflect similar underlying temporal processes and mechanisms (Block, 1990). In the production task, a participant has to produce an interval equal to an interval previously reported (i.e., "Produce 2 seconds"). In the verbal estimation task, after experiencing target duration, the participant has to translate this subjective duration into clock units. An adapted version of experiments utilizing these tasks can be downloaded from the GitHub repository of this book. These two methods involve the comparison of the experienced duration with internal information concerning conventional duration units, such as milliseconds and seconds (i.e., reference memory; Zakay, 1990). In both cases, a translation from an objectively labeled duration to a subjectively experienced duration (i.e., time production) and vice versa (i.e., verbal estimation) has to be completed. Time production and verbal estimation are appropriate ways for investigating individual differences related to the internal clock (its speed rate or variables influencing it), given that they best 
reflect the speed or rate of functioning of an internal clock (Baudouin et al., 2006; Glicksohn, \& Hadad, 2011).

In the time reproduction tasks, participants are required to reproduce the duration of the temporal interval previously presented. The task is composed of two phases: the encoding and the reproduction phase. First, participants experience the target duration (i.e., encoding phase), and then they are asked to delimit a time interval (by pressing a designed key) equivalent to the target duration previously presented (i.e., reproduction phase; Mioni, Stablum, McClintock, \& Grondin, 2014c). An adapted version of such experiments can be downloaded from the GitHub repository of this book. Compared to the production or verbal estimation tasks, a reproduction task is used less in the investigation of individual differences at the internal clock level. In fact, the speed rate of the internal clock is the same when experiencing the target duration and when reproducing it. This method, which relies on a comparison of previously experienced temporal intervals, has the potential disadvantage that it might be mainly an index of the consistency of the subjective time experience, providing no information about variation at the pacemaker rate. Even if the rate of physiological and cognitive processes varies, the same rate will subserve a person's experience of the target duration (encoding phase) and its reproduction (reproduction phase). Thus, the reproduction method may detect individual differences only if it is used in the framework of psychophysical studies, in which duration is varied. In addition, judgments obtained by using the reproduction method (as well as the production method) may be confounded by extraneous variables such as the desire to terminate the experiment sooner, impatience, or the inability to delay a response.

Researchers often use these three methods interchangeably without providing an explanation for the selection of a given method. It is important to remember that each method activates different timing-related processes and presents some specific perceptual errors. For example, participants tested with the verbal estimation method are prone to respond to the estimated duration using rounded numbers and data are often characterized by a great amount of variability compared to the other methods (Grondin, 2010; Zakay, 1990). Time reproduction is considered to be more accurate and reliable than time production and verbal estimation; however, it is less useful for investigating variations in the pacemaker rate. Time production and verbal estimation show more inter-participant variability than time reproduction, but can be successfully used in studies where the rate of the internal pacemaker is manipulated.

Moreover, it is important to consider that each method requires cognitive resources to be processed. In fact, a reproduction task recruits attention and working memory to keep active the reference duration in order to subsequently 
reproduce it. Conversely, the production and verbal estimation tasks require other cognitive processes such as language processing and access to long-term memory (Block, Zakay, \& Hancock, 1998). Time production and reproduction tasks require a motor action ${ }^{1}$ to produce and reproduce, respectively, the temporal intervals after the presentation of a given target duration.

\section{$3 \quad$ Scoring Methods}

Data collected from the reproduction, production, and verbal estimation tasks may be scored in term of the: $(1) \mathrm{S}_{\mathrm{d}} / \mathrm{O}_{\mathrm{d}}$ ratio, (2) absolute discrepancy $\left(\left|\mathrm{S}_{\mathrm{d}}-\mathrm{O}_{\mathrm{d}}\right|\right),(3)$ absolute error $\left(\left|\mathrm{S}_{\mathrm{d}}-\mathrm{O}_{\mathrm{d}}\right| / \mathrm{O}_{\mathrm{d}}\right)$, and (4) coefficient of variation $\left(\mathrm{sD}^{2} /\right.$ $S_{d}$; Glicksohn \& Hadad, 2012; Mioni et al., 2014). $S_{d}$ represents the subjective duration expressed by the participants and $\mathrm{O}_{\mathrm{d}}$ represents the objective target duration presented.

In computing the $S_{d} / O_{d}$ ratio, the time estimation is expressed in terms of proportion of physical duration and then the results are compared across different temporal intervals. The absolute discrepancy $\left(\left|\mathrm{S}_{\mathrm{d}}-\mathrm{O}_{\mathrm{d}}\right|\right)$ reflects the magnitude of temporal error without giving any information regarding the duration (i.e., over- or under-estimation). The absolute error $\left(\left|\mathrm{S}_{\mathrm{d}}-\mathrm{O}_{\mathrm{d}}\right| / \mathrm{O}_{\mathrm{d}}\right)$ may be the most sensitive of all indices and reflects a more generalized disruption of timing (Brown \& Boltz, 2002). Finally, the coefficient of variance (Cv) is an index of timing variability over a series of trials.

Interestingly, Glicksohn and Hadad (2012) tried to compare and contrast these different indices (with exclusion of $\mathrm{CV}$ ) while studying sex difference in time perception. The authors only considered the time production task, but the results are a good starting point for extending the conclusions to the time reproduction and verbal estimation methods. Briefly, the results showed that when $S_{d} / O_{d}$ ratio is considered, female participants had a lower mean ratio in comparison to the male participants. In contrast, results reported using $\left|\mathrm{S}_{\mathrm{d}}-\mathrm{O}_{\mathrm{d}}\right|$ and $\left|\mathrm{S}_{\mathrm{d}}-\mathrm{O}_{\mathrm{d}}\right| / \mathrm{O}_{\mathrm{d}}$ indices were seriously compromised by wide individual differences. Regarding the $\mathrm{CV}$, it is an interesting index when comparing clinical and healthy groups and when group and individual variability is under investigation. With clinical population, higher temporal variability is a robust finding (Cester, Mioni, \& Cornoldi, 2017; Jones \& Jahanshahi, 2014; Mioni,

1 Verbal speech can also be considered a motor action but here we refer as "motor action" only the context of physical motor action. It includes the preparation and the execution of a physical movement.

$2 \mathrm{SD}=$ standard deviation. 
Grondin, \& Stablum, 2014a; Mioni, Stablum, Prunetti, \& Grondin, 2016b) and it has been explained as a manifestation of difficulties in maintaining a stable representation of duration. This difficulty is accentuated in patients due to attentional, working memory, and executive dysfunction, and could be viewed as a manifestation of failure to fully attend to temporal information (Cester et al., 2017; Jones \& Jahanshahi, 2014; Mioni, Grondin, \& Stablum, 2014a; Mioni, Stablum, Prunetti, \& Grondin, 2016b).

What are the benefits of one measure over the other? If multiple temporal intervals are included within the same experimental design $\mathrm{S}_{\mathrm{d}} / \mathrm{O}_{\mathrm{d}}$ ratio should be used. This index also provides information regarding the direction of duration (over- or under-estimation); if an index of amount of error is needed, the absolute discrepancy $\left(\left|S_{d^{-}} O_{d}\right|\right)$ is more appropriate. The absolute error $\left(\mid S_{d^{-}}\right.$ $\left.\mathrm{O}_{\mathrm{d}} / / \mathrm{O}_{\mathrm{d}}\right)$ includes a mix of the other two indices reflecting a more generalized disruption of timing. The CV can be included if different groups (i.e., clinical vs. healthy or children vs. adults) are compared and it is an interesting measure of temporal variability.

Taking into consideration that each method activates different time-related processes (attentional or working memory resources) and activate different representation of time (subjective or objective), one way to select the appropriate method is to take into account other variables involved such as the temporal interval, the motor component, cognitive load, and cognitive strategies as well as the group of participants under investigation. A general consideration is also that, given the tendency we have to round off time estimates with chronometric units, verbal estimations produce more variability and are less accurate (greater discrepancy with respect to the standard duration) than time production and reproduction methods.

\subsection{Temporal Range}

Regarding the temporal range, a general tendency in timing literature, mainly in neuroscience researchers, is to emphasize a distinction between intervals above and below 1s, which is based on differential pharmacological effects (Rammsayer, 2008) and on patient studies with various cases of brain damage (see Allman \& Meck, 2012; Meck, 2005; Mioni, Grondin, \& Stablum, 2014a; Piras et al., 2014). Moreover, researchers claim that the processing of smaller intervals is more sensory-based or benefits from some automatic processing, whereas the processing of longer intervals requires the support of cognitive 
resources (also see Lewis \& Miall, 2003; Mioni, Stablum, \& Grondin, 2014b). Even if this ' 1 's' transition period remains somewhat arbitrary, there is certainly some turning point on the time continuum. This is evident considering the benefit observed from adopting explicit counting strategy for processing long temporal intervals (around seconds range) with respect to brief temporal interval (around milliseconds range) (Grondin, Meilleur-Wells, \& Lachance, 1999; Grondin, Ouellet, \& Roussel, 2004; Mioni, Stablum, \& Grondin, 2014b). Indeed, there are empirical reasons to believe that this transition occurs around $1200 \mathrm{~ms}$. Using a time discrimination task, ${ }^{3}$ Mioni and colleagues (2014) tested participants with duration ranging from 400 to $1600 \mathrm{~ms}$ to investigate if temporal discrimination is influenced by the temporal range and/or by the context. ${ }^{4}$ Results suggest that context influences time discrimination performance only when the temporal range under investigation is below $1300 \mathrm{~ms}$ and the temporal intervals varied within blocks. In the case of temporal intervals longer than $1300 \mathrm{~ms}$, participants presented a tendency to respond "long" independently of the procedure used. Researchers claimed that processing of smaller intervals is more sensory based, or benefits from some automatic processing, whereas the processing of longer intervals requires the support of cognitive resources (Hellström \& Rammsayer, 2004; Lewis \& Miall, 2003). This is also confirmed by results observed from patients' studies with brain lesions (see later sections in this chapter and also Allman \& Meck, 2012; Jones \& Jahanshahi, 2014; Mioni, Grondin, \& Stablum, 2014a; Piras et al., 2014).

Therefore, if the theoretical question involves exploring "pure" temporal abilities, without (or with reduced) influence of cognitive processes, brief temporal intervals (below 1200-1300 ms) should be privileged.

\subsection{Concurrent Secondary Task}

In most cases, when performing temporal tasks, participants are also engaged in an additional exercise, called the "secondary task". The aim of adding a secondary task is to: (1) avoid counting strategies that are often used when experiencing long temporal intervals (see Section 4.3) and (2) understand the effects of cognitive load on duration judgments. As mentioned before, attention plays a major role in prospective duration experience. The Attentional-Gate Model (Block \& Zakay, 1996; Zakay \& Block, 1996) proposes that the

3 In a time discrimination task, participants are required to judge the relative duration of two time intervals presented successively and indicate whether the second stimulus is presented longer or shorter with respect to the first one.

4 The context effect here refers to the presentation of temporal intervals randomized within or across blocks (see also Jones and McAuley 2005). 
experienced duration of a time period depends on the amount of information encoded during the temporal information processor and by a non-temporal information processor. Task demands (variation in the cognitive load) determine the way in which a person divides attention between the two processors. In fact, employing a concurrent secondary task gives the opportunity of testing the effects of variation of cognitive resources on temporal processing (Block, Hancock, \& Zakay, 2010). It is thought that when a person is working on a difficult or attention-demanding task, time seems to pass quickly, but if a person is working on an easy or less attentional-demanding task, time seems to pass slowly. Therefore, time processing is adversely affected by the attentional or workload demands of any non-temporal (secondary) task (Brown, 1997, 2008; Brown \& Boltz, 2002; Zakay \& Block, 2004). It is possible to test the effects of the secondary task by: (1) manipulating the cognitive load of the secondary task by employing easy or more complex secondary tasks (Brown, 1985, 1997), or (2) varying the instruction by asking participants to focus attention mainly on the temporal task, or on the secondary task or both.

Generally, dual-task conditions (temporal task + secondary task) typically cause time judgments to become less accurate than single-task conditions in which participants judge time alone, but the effects also vary depending on the temporal task used. Verbal estimates and reproductions usually decrease (shorter temporal intervals estimated or reproduced) with greater processing demands during a time period, the reverse usually holds for time productions. During time reproduction tasks, temporal reproductions are longer if the timing task is defined as the primary task (attention fully focused on performing the timing task) than if it is defined as the secondary task (attention is focused on the secondary task).

\subsection{Counting during Timing Tasks}

Adding a secondary task during the temporal task, not only gives the opportunity to test variations of attention and cognitive resources on time processing, but it also prevents the participants from adopting strategies, such as counting, during time processing (Grondin et al., 1999, 2004). It has been demonstrated that explicit counting improves temporal sensitivity in children as well as in adults (Clément \& Rattat, 2006) and also in clinical groups (Perbal, Couillet, Azouvi, \& Pouthas, 2003; Perbal, Couillet, Azouvi, \& Pouthas, 2005). An explanation of this counting advantage, rely on the knowledge that segmenting information into smaller parts helps the processing of this information. Moreover, counting reduces the contribution of memory to the overall variance in the timing process. This reduction is made possible by the remembering of the number counted rather than by remembering an interval representation. 
However, letting participants use strategies during the timing task interferes with the meaning of testing "time". Therefore, it is preferred to not allow participants in adopting counting or any other strategy. One option is to employ brief temporal intervals in which counting is not advantageous (Grondin et al., 1999, 2004). Grondin (2004) showed, in a time discrimination task, benefits from explicit counting at $1.6 \mathrm{~s}$, but not at $.8 \mathrm{~s}$, when temporal intervals were marked by auditory as well as visual stimuli (see also Mioni, Stablum, \& Grondin, 2014b; Mioni et al., 2016a). Rattat and Droit-Volet (2012) compared the effects of the three classic methods to avoid counting: (1) instructions not to count, (2) articulatory suppression, and (3) administration of an interference task in temporal generalization, time bisection, and reproduction tasks with two duration ranges (i.e., $1-4 \mathrm{~s}$ and $2-8 \mathrm{~s}$ ). Results showed that all three no-counting conditions prevented participants from counting, and interestingly, the instructions not to count actually constituted the simplest and more efficient method of preventing counting in timing tasks. An alternative way to prevent participants from counting is to employ a motor task (i.e., continuous finger tapping). This method has the advantage to be employed independently of the modality used to present the temporal intervals. In some cases using a counting or articulatory suppression strategies with auditory stimuli might create and additional modality interference (Mioni et al., 2016a).

\subsection{Methods for Producing and Reproducing Time}

In the case of time production and reproduction, an additional issue concerns the motor action required to produce or reproduce temporal intervals. Participants need to integrate their motor action in order to produce a precise button press to process the temporal interval (Droit-Volet, 2010; Mioni et al., 2014). Moreover, preparing and executing a motor action requires cognitive resources that might result in additional variance (Caldara et al., 2004). For instance, we can assume that people suffering from deficits in planning, preparation, and execution of motor movements could have poor temporal performance in a temporal reproduction task due to their motor-related deficits rather than to their inability to estimate time (Bloxham, Dick, \& Mooret, 1987; Stuss et al., 1989). Mioni and colleagues (2014) compared the effects of three classical methods used to reproduce time with temporal intervals ranging from 1 to $18 \mathrm{~s}$ : (1) pressing a designed key at the end of the reproduction, (2) pressing a designed key to start and stop the reproduction, and (3) continuous pressing a designed key to reproduce the duration. Results showed that temporal performance depends on the method employed and on the interval range under investigation. In fact, the second method (i.e., pressing to start and end the reproduction) led to better accuracy $\left(\left|\mathrm{S}_{\mathrm{d}}-\mathrm{O}_{\mathrm{d}}\right|\right)$ than did 
Methods 1 and 3 with longer durations. However, when short durations were employed (i.e., $1 \mathrm{~s})$, method 2 generated a higher absolute discrepancy $\left(\mid \mathrm{S}_{\mathrm{d}^{-}}\right.$ $\mathrm{O}_{\mathrm{d}} \mid$ ), indicating lower performance. Participants using method 1 showed good performance only when reproducing 1-s intervals. Of interest are the results obtained with CVs. Although participants using method 3 showed the lowest level of accuracy $\left(\left|\mathrm{S}_{\mathrm{d}}-\mathrm{O}_{\mathrm{d}}\right|\right)$ and under-reproduced temporal durations $\left(\left|\mathrm{S}_{\mathrm{d}} / \mathrm{O}_{\mathrm{d}}\right|\right)$ more than participants using method 1 or 2 , method 3 led to the lowest CVs. Interestingly, pressing continuously to reproduce the duration, generated less variability than the other methods, in particular when participants reproduce longer durations.

\subsection{Modality and Stimulus Type}

Finally, when building a timing task the modality and the type of temporal interval should be taken into account. The modality refers to the sensory modality used to present the temporal intervals (visual, auditory, or tactile). It is known that time perception is influenced by the sensory modality used for marking the time intervals (Grondin, 2003). Timing is more precise when stimuli are presented in the auditory rather than the visual modality (Grondin, 2003, 2010; Ulrich, Nitschkle, \& Rammsayer, 20o6), and this auditory superiority might be due to the automaticity of temporal processing in audition.

Also, the way the temporal intervals are presented influence temporal performance. Temporal intervals can be defined as "filled" or "empty". An interval is defined "filled" when there is one given continuous signal between onset and offset; the signals' onset and offset marks the interval's beginning and end. An "empty" interval is a silent duration, that is, without stimulation, included within two sensory signals that mark the beginning and end of the interval. Generally, duration estimates lengthen if duration is filled as opposed to unfilled. However, the differences between performance with filled and empty intervals are used depends on the type of markers, on the range of duration investigated, and the method employed (Grondin, 2008, 2010).

\section{5}

\section{Prospective Timing in Clinical Populations}

It is useful to bear in mind that there is no human clinical condition that can be defined solely as a disorder of timing and time perception per se. However, distortions in ones' timing ability are present, to varying degrees, in many patient populations, and may or may not accompany differences in other aspects of sensory processing, as well as developmental, cognitive, and behavioural profiles. From a clinical perspective, examinations of the timing 
ability in patients with certain psychiatric or behavioural disorders may help to ameliorate understanding of the psychological experience of these disorders and their potential remediation. In this regard, temporal distortion in Parkinson's patients (PD) and traumatic brain injury patients (TBI) will be discussed. The selection of these two clinical groups concerns the presentation of two different patterns of temporal dysfunction and will help in the understanding of the source of their temporal misperception. Readers who are more interested in psychopathologies will also find a large number of time-related articles in the neuropsychological or psychiatric literature (Allman \& Meck, 2012; Meck, 2005): Developmental findings in healthy (Droit-Volet, 2013) and clinical children (Autism: Allman \& Falter, 2015; Attention-deficit hyperactivity disorder: Hart, Radua, Mataix-Cols, \& Rubia, 2012; Toplak, Dockstader, \& Tannock, 2006), depressed (Thönes, \& Oberfeld,2015) and anxious (Mioni et al., 2016a) patients; patients with schizophrenia (Thoenes \& Oberfeld, 2017).

\subsection{Timing in Parkinson's Patients}

$\mathrm{PD}$ is a movement disorder characterized by bradykinesia, tremor, rigidity, and postural instability. Disease symptoms occur as a result of selective, progressive, and chronic degeneration of the nigrostriatal and mesocorticolimbic dopamine systems (Allman \& Meck, 2012; Jankovic \& Tolosa, 2007; Meck, 2005). Dysfunctions in dopamine transmission have also been identified as the main cause of temporal impairment in PD patients. Evidence suggests that PD patients show dysfunctions in time perception in various temporal tasks (Jones \& Jahanshahi, 2014).

PD patients have been tested due to their basal ganglia dysfunction and dopaminergic alteration. Dopaminergic antagonists produce a deceleration of the subjective clock speed (Buhusi \& Meck, 2005). These results traditionally elected the dopaminergic system and the basal ganglia as a "house" of the pacemaker-clock system. However, the pathophysiology of PD is complex and evolves in the course of the illness. The basal ganglia seem to be particularly involved in timekeeping functions, and a strict connection is hypothesized between the basal ganglia and cortical structures supporting conscious representation and memory for time. The frontal cortex, mainly the dorsolateral prefrontal cortex, has an established role in a range of cognitive processes, such as working memory and decision-making, which the clock model proposes as necessary for efficient interval timing. Therefore, it can be difficult in PD patients to tease apart whether the timing difficulties are driven by a core timing dysfunction, or by the disruption of general cognitive processes.

When investigating perceptual timing in patients with movement disorders the most effective tasks are those not involving reaction time and responses 
based on motor components. Time discrimination and time bisection tasks fit these criteria; however, studies have been conducted also with time production, verbal estimation, and time reproduction tasks. Despite the motor limitation (that can be controlled including a simple motor task), results from studies conducted with time reproduction tasks showed both increased and reduced variability and over- and under-reproduction in PD patients compared to controls. It is possible that some of the contradictory findings reported in the literature on timing and time perception with PD patients may be explained by differences in temporal range and stimuli modality.

An interesting phenomenon that is often observed is the "migration effect" (Malapani, Deweer, \& Gibbon, 2002; Malapani et al., 1998). When temporal intervals in different temporal ranges are used within the same block, short intervals are over-reproduced and long intervals are under-reproduced (see also the Vierordt's law; Lejeune, \& Wearden, 2009). What is the source of this effect in PD patients? Malapani et al. (2002) suggested that the memory for learned durations is the source of the temporal impairment rather than variation at the clock level.

Some other critical factors that have to be taken into account when testing PD patients is the effect of medication and the role of cognitive factors. Regarding the first point, Jones and Jahanshahi (2014) reported that half of the studies that reported a direct comparison between "ON" and "OFF" medication indicated a beneficial effect of medication on perceptual timing. However, some studies have also reported the opposite findings, reflecting a negative effect of dopaminergic remediation of relatively preserved basal ganglia circuits. Moreover, patients can vary in severity and duration of the illness, which are factors that can mediate the medication impact (Merchant et al., 2008).

Finally, regarding the involvement of cognitive function on temporal processing, this leads to an important area of debate. Does the temporal dysfunction observed in PD patients reflect a dysfunction in critical timing regions or it is mediated by global cognitive impairment? Traditionally, the way for testing the extent to which cognitive impairment is correlated with timing performance is to compare performance in neuropsychological and temporal tasks. Even though most studies report a complete neuropsychological evaluation (Jones \& Jahanshahi, 2014), very few studies run correlational analyses. Jones et al. (2008) conducted a factor analysis and reported a common factor between measures of attention and time production that was distinct from time reproduction. However, Merchant et al. (2008) found that performance on a range of cognitive tasks did not discriminate those with PD who did well or poorly on a range or motor and perceptual timing tasks. Importantly, in all these studies, the group of PD patients was treated as a whole sample, however, 
different levels of impairment exist at the individual level. A different approach was followed by Mioni et al. $(2015,2017)$. That is, by using Litvan's criteria (Litvan et al., 2012), patients with a diagnosis of PD who failed at least two tasks (below two standard deviations) in the same domain or in different domains were defined as PD with mild cognitive impairment (MCI) and tested separately from PD patients without MCI. Results showed that PD-MCI patients were less accurate and more variable with respect to PD-no-MCI and controls that demonstrated similar temporal abilities. It is, therefore, evident, that the level of cognitive function can influence temporal performance in PD patients, but this does not preclude that "pure" clock dysfunction is also present.

\subsection{Time in Traumatic Brain Injury Patients}

Neuropathological evidence suggest a marked heterogeneity of injuries across TBI patients. However, it is apparent that diffuse axonal injury is common, and that damage occurs most frequently in the frontal and temporal lobes. Temporal impairments in patients with твг are expected considering the disruption of cognitive function involved in temporal processing (Mioni, Grondin, \& Stablum, 2014a; Piras et al., 2014).

Reviewing the studies conducted to investigate temporal dysfunction in тв I patients (Mioni, Grondin, \& Stablum, 2014a), four used a time reproduction task (Meyers \& Levin, 1992; Mioni, Stablum, McClintock, \& Cantagallo, 2012; Mioni, Mattalia, \& Stablum, 2013a; Perbal et al., 2003), three a verbal estimation task (Anderson \& Schmitter-Edgecombe, 2011; Meyers \& Levin, 1992; Schmitter-Edgecombe \& Rueda, 2008), two a time production task (Mioni, Mattalia, \& Stablum, 2013a; Perbal et al., 2003), and two a time discrimination task $^{5}$ (Mioni, Mattalia, \& Stablum, 2013a; Mioni, Stablum, \& Cantagallo, 2013b).

The studies conducted with the time reproduction task showed that тві patients were as accurate as controls $\left(\mathrm{S}_{\mathrm{d}} / \mathrm{O}_{\mathrm{d}}\right.$ ratio $)$ and showed higher variability (CV) in their timing judgments, indicating dysfunction in maintaining a stable representation of temporal intervals. In the study conducted by Perbal et al. (2003), participants were also asked to perform a secondary (non-temporal) task together with the time reproduction task. Similar under-reproduction was observed in твI patients and controls in both simple (time reproduction only) and concurrent (time reproduction + non-temporal task) conditions, in particular under the latter condition.

5 In time discrimination tasks, participants are required to compare the relative duration of two intervals that are sequentially presented (standard-comparison) and then judge, which one was longer or shorter. Since this chapter does not cover this method, please refer to the original papers for a detailed presentation of the results (Mioni et al. 2013a, 2013b). 
The studies conducted with a time production task confirmed and extended the results obtained with the time reproduction task. Again, твI patients were as accurate as controls $\left(\mathrm{S}_{\mathrm{d}} / \mathrm{O}_{\mathrm{d}}\right.$ ratio $)$ and showed higher temporal variability (Cv; Mioni et al., 2013b; Perbal et al., 2003). Regarding the impact of a concurrent non-temporal task, no effect was found (time production only vs. time production + non-temporal task) and this finding applies to both groups (i.e., твI and controls). твIs and controls showed the same performances $\left(\mathrm{S}_{\mathrm{d}} / \mathrm{O}_{\mathrm{d}}\right.$ ratio and $\left.\mathrm{Cv}\right)$ in both simple and concurrent conditions (Perbal et al., 2003).

Three studies were conducted with a verbal estimation task but performance was only analysed in two of them. Indeed, in Meyers and Levin's (1992) study, performance at a verbal estimation task was not analysed due to the extreme variability noted in the тві sample. Schmitter-Edgecombe and Rueda (2008), as well as Anderson and Schmitter-Edgecombe (2011), reported lower accuracy $\left(\left|\mathrm{S}_{\mathrm{d}}-\mathrm{O}_{\mathrm{d}}\right|\right)$, higher under-estimation $\left(\mathrm{S}_{\mathrm{d}} / \mathrm{O}_{\mathrm{d}}\right.$ ratio $)$, and more variability (i.e., $\mathrm{CV}$ ) in TBI patients than controls.

In brief, TBI patients and controls showed similar performances $\left(\left|\mathrm{S}_{\mathrm{d}^{-}} \mathrm{O}_{\mathrm{d}}\right|\right.$ or $\mathrm{S}_{\mathrm{d}} / \mathrm{O}_{\mathrm{d}}$ ratio) when time reproduction and time production tasks are employed. However, твI patients performed less accurately than controls when verbal estimation was used. Moreover, in all studies, variability is higher with твI patients than with controls.

In sum, the revision of the existing literature investigating time perception in твI patients showed that temporal dysfunctions in TBI patients were related to deficits in cognitive function involved in temporal processing such as working memory, attention, and executive function rather than an impairment in time estimation per se. In fact, temporal dysfunctions were observed when the tasks employed required high cognitive functions to be performed (Mioni, Mattalia, \& Stablum, 2013a; Mioni, Stablum, \& Cantagallo, 2013b). The consistent higher temporal variability observed is a sign of impaired frontally mediated cognitive functions that affect temporal representation.

This chapter provided a general overview of the typical perceptual timing tasks used under the prospective paradigm. The tasks that have been described were the time production, verbal estimation, and time reproduction tasks. Each of these tasks highlights different temporal characteristics and requires different cognitive processes. Briefly, time production and verbal estimation tasks are suitable methods for investigating individual differences in the speed rate of 
the internal clock, while time reproduction is useful if it is used in the framework of psychophysical studies where duration is varied.

Different factors should be taken into account when selecting a timing task. First of all, the temporal range is critical. Brief temporal ranges should be preferred to reduce additional cognitive factors that are required when performing long temporal intervals (i.e., attention and working memory). Moreover, a motor component can alter temporal performance in time production and time reproduction, when brief temporal intervals are employed. If longer temporal intervals are used (around $1.2 \mathrm{~s}$ ), participants have the natural tendency to use strategies (i.e., counting) during the temporal task. Segmenting a long temporal interval using counting improves temporal performance, however in this case the results can be less interpreted as an index of temporal ability and more as an index of counting. To prevent participants from counting, various methodologies can be used, and simply asking participants not to count has been demonstrated as a good method to eliminate counting. Finally, this chapter briefly presented a review of the main findings observed in timing literature when PD and TBI patients are tested with some specific clinical and methodological suggestions.

\section{References}

Allman, M., \& C. Falter (2015). Abnormal timing and time perception in autism spectrum disorder? A review of the evidence. In Vatakis, A., \& M.J. Allman (Eds.). Time distortions in mind-temporal processing in clinical populations (pp. 37-56). Leiden, The Netherlands: Brill Academic Publishers

Allman, M.J., \& W.H. Meck (2012). Pathophysiological distortions in time perception and timed performance. Brain, $135,656-677$.

Anderson, J.W., \& M. Schmitter-Edgecombe (2011). Recovery of time estimtion following moderate to severe traumatic brain injury. Neuropsychology, 25, 36-44.

Baudouin, A., S. Vanneste, M. Isingrini, \& V. Pouthas (2006). Differential involvement of internal clock and working memory in the production and reproduction of duration: A study on older adults. Acta Psychologica, 121, 285-296.

Block, R.A. (1990). Models of psychological time. In Block, R.A. (Ed.), Cognitive models of psychological time (pp. 1-35). Hillsdale, NJ: Lawrence Erlbaum Associates Inc.

Block, R.A., \& D. Zakay (1996). Models of psychological time revised. In Helfrich, H. (Ed.), Time and mind (pp. 171-195). Kirkland, wa: Hogrefe and Huber.

Block, R.A., D. Zakay, \& P.A. Hancock (1998). Human aging and duration judgments: A meta-analytic review. Psychology \& Aging, 13, 584-596. 
Block, R.A., P.A. Hancock, \& D. Zakay (2010). How cognitive load affects duration judgments: A meta-analytic review. Acta Psychologica, 134, 330-343.

Bloxham, C.A., D.J. Dick, \& J.M. Mooret (1987). Reaction times and attention in Parkinson's disease. Journal of Neurology, Neurosurgery, and Psychiatry, 50, 1178-1183.

Brown, S.W. (1985). Time perception and attention: The effects of prospective versus retrospective paradigms and task demands on perceived duration. Perception \& Psychophysics, 38(2), 115-124.

Brown, S.W. (1997). Attentional resources in timing: Interference effects in concurrent temporal and nontemporal working memory tasks. Perception \& Psychophysics, 59, 1118-1140.

Brown, S.W. (2008). Time and attention: Review of the literature. In Grondin, S. (Ed.), Psychology of Time (pp. 111-138). Bingley, England: Emerald.

Brown, S.W., \& M.G. Boltz (2002). Attentional processes in time perception: Effects of mental workload and event structure. Journal of Experimental Psychology: Human Perception and Performance, 28, 600-615.

Buhusi, C.V., \& W.H. Meck (2005). What makes us stick? Functional and neural mechanisms of interval timing. Nature Review Neuroscience, 6, 755-765.

Caldara, R., M.-P. Deiber, C. Andrey, G.M. Michel, G. Thut, \& C.-A. Hauert (2004). Actual and mental motor preparation and execution: A spatiotemporal ERP study. Experimental Brain Research, 159, 389-399.

Cester, I., G. Mioni, \& C. Cornoldi (2017). Time processing in children with mathematical difficulties. Learning and Individual Differences, 58, 22-30.

Clément, A., \& S. Droit-Volet (2006). Counting in a time discrimination task in children and adults. Behavioural Processes, 71, 164-171.

Droit-Volet, S. (2010). Stop using time reproduction tasks in a comparative perspective without further analyses of the role of the motor response: The example of children. European Journal of Cognitive Psychology, 22(1), 130-148.

Droit-Volet, S. (2013). Time perception in children: A neurodevelopmental approach. Neuropsychologia, 51(2), 220-234.

Droit-Volet, S., \& W.H. Meck (2007). How emotions colour our perception of time. Trends in Cognitive Sciences, $11(12), 504-513$.

Fraisse, P. (1984). Perception and estimation of time. Annual Review of Psychology, 35, $1-36$.

Gibbon, J. (1977). Scalar expectancy theory and Weber's law in animal timing. Psychological Review, 84, 279-385.

Gibbon, J., R.M. Church, \& W.H. Meck (1984). Scalar timing in memory. In Gibbon, J. \& L.G. Allan (Eds.), Timing and time perception (pp. 52-77). New York: The New York Academy of Science.

Glicksohn, J., \& Y. Hadad (2011). Sex differences in time production revisited.Journal of Individual Differences, 33(1), 35-42. 
Grondin, S. (2003). Sensory modalities and temporal processing. In Helfrich, H. (Ed.), Time and mind II (pp. 61-77). Göttingen: Hogrefe \& Huber.

Grondin, S. (2008). Methods for studying psychological time. In Grondin, S. (Ed.), Psychology of time (pp. 51-74). Bingley, Uk: Emerald Group Publishing.

Grondin, S. (2010). Timing and time perception: A review of recent behavioral and neuroscience findings and theoretical directions. Attention, Perception \& Psychophysics, $72(3), 561-582$.

Grondin, S., G. Meilleur-Wells, \& R. Lachance (1999). When to start explicit counting in a time-intervals discrimination task: A critical point in the timing process of humans. Journal of Experimental Psychology: Human, Perception and Performance, 25, 993-1004.

Grondin, S., B. Ouellet, \& M.-E. Roussel (2004). Benefits and limits of explicit counting for discriminating temporal intervals. Canadian Journal Experimental Psychology, $58,1-12$.

Hart, H., J. Radua, D. Mataix-Cols, \& K. Rubia (2012). Meta-analysis of fMRI studies of timing in attention-deficit hyperactivity disorder (ADHD). Neuroscience \& Biobehavioral Reviews, 36(10), 2248-2256.

Hellström, Á., \& T.H. Rammsayer (2004). Effects of time-order, interstimulus interval, and feedback in duration discrimination of noise bursts in the $5^{0-}$ and 1000-ms ranges. Acta Psychologica, 116, 1-20.

Jankovic, J., \& E. Tolosa (Eds.) (2007). Parkinson's disease and movement disorders. Philadelphia: Lippincott Williams

Jones, C.R.G., \& M. Jahanshahi (2014). Motor and perceptual timing in Parkinson's disease. In Merchant, H. \& V. de Lafuente (Eds.), Neurobiology of interval timing. Advances in experimental medicine and biology (pp. 265-290). New York: Springer Verlag.

Jones, M.R., \& J.D. McAuley (2005). Time judgments in global temporal contexts. Perception \& Psychophysics, 67, 398-417.

Jones, C.R.G., T.J. Malone, G. Dirnberger, M. Edwards, \& M. Jahanshahi (2008). Basal ganglia, dopamine and temporal processing: Performance on three timing tasks on and off medication in Parkinson's disease. Brain \& Cognition, 68(1), 30-41.

Karaminis, T., G.M. Cicchini, L. Neil, G. Cappagli, D. Aagten-Murphy, D. Burr, \& E. Pellicano (2016). Central tendency effects in time interval reproduction in autism. Scientific Reports, 28(6), 28570.

Lejeune, H., \& J.H. Wearden (2009). Vierordt's the experimental study of the time sense (1868) and its legacy. European Journal of Cognitive Psychology, 21, 941-960.

Lewis, P.A., \& R.C. Miall (2003). Distinct systems for automatic and cognitively controlled time measurement: Evidence from neuroimaging. Current Opinion in $\mathrm{Neu}-$ robiology, 13, 250-255. 
Litvan, I., J.G. Goldman, A.I. Tröster, B.A. Schmand, D. Weintraub, R.C. Petersen, B. Mollenhauer, C.H. Adler, K. Marder, C.H. Williams-Gray, D. Aarsland, J. Kulisevsky, M.C. Rodriguez-Oroz, D.J. Burn, R.A. Barker, \& M. Emre (2012). Diagnostic criteria for mild cognitive impairment in Parkinson's disease: Movement disorder society task force guidelines. Movement Disorders, 27(3), 349-356.

Malapani, C., B. Rakitin, R. Levy, W.H. Meck, B. Deweer, B. Dubois, \& J. Gibbon (1998). Coupled temporal memories in Parkinson's disease: A dopamine-related dysfunction. Journal of Cognitive Neuroscience, $10(3), 316-331$

Malapani, C., B. Deweer, \& J. Gibbon (2002). Separating storage from retrieval dysfunction of temporal memory in Parkinson's disease. Journal of Cognitive Neuroscience, 14(2), 311-322.

Meck, W.H. (2005). Neuropsychology of timing and time perception. Brain \& Cognition, 58, 1-8.

Merchant, H., M. Luciana, C. Hooper, S. Majestic, \& P. Tuite (2008). Interval timing and Parkinson's disease: Heterogeneity in temporal performance. Experimental Brain Research, 184(2), 233-248.

Meyers, C.A., \& H.S. Levin (1992). Temporal perception following closed head injury: Relationship of orientation and attention span. Neuropsychiatry, Neuropsychology, and Behavioral Neurology, 1, 28-32.

Mioni, G., F. Stablum, M.S. McClintock, \& A. Cantagallo (2012). Time-based prospective memory in severe traumatic brain in jury patients: The involvement of executive functions and time perception. Journal of International Neuropsychology Society, 18 , 697-705.

Mioni, G., G. Mattalia, \& F. Stablum (2013a). Time perception in severe traumatic brain injury patients: A study comparing different methodologies. Brain \& Cognition, 81 , 305-312.

Mioni, G., F. Stablum, \& A. Cantagallo (2013b). Time discrimination in traumatic brain injury patients. Journal of Clinical Experimental Neuropsychology, 35, 90-102.

Mioni, G., S. Grondin, \& F. Stablum (2014a). Temporal dysfunction in traumatic brain injury patients: Primary or secondary impairment? Frontiers in Human Neuroscience, 8(269).

Mioni, G., F. Stablum, \& S. Grondin (2014b). Interval discrimination across different duration ranges with a look at spatial compatibility and context effects. Frontiers in Psychology, 5(717).

Mioni, G., F. Stablum, S.M. McClintock, \& S. Grondin (2014c). Different methods for reproducing time, different results. Attention, Perception \& Psychophysics, 76, 675-681.

Mioni, G., L. Meligrana, S. Grondin, F. Perini, L. Bartolomei, \& F. Stablum (2015). Effects of emotional facial expression on time perception in patients with Parkinson's disease. Journal of the International Neuropsychological Society, 22(9), 890-899. 
Mioni, G., M. Grassi, V. Tarantino, F. Stablum, S. Grondin, \& P.S. Bisiacchi (2016a). The impact of a concurrent motor task on auditory and visual temporal discrimination tasks. Attention, Perception \& Psychophysics, 78, 742-748.

Mioni, G., F. Stablum, E. Prunetti, \& S. Grondin (2016b). Time perception in anxious and depressed patients: A comparison between time reproduction and time production tasks. Journal of Affective Disorders, 196, 154-163.

Mioni, G., S. Grondin, L. Meligrana, F. Perini, L. Bartolomei, \& F. Stablum (2017). Effects of happy and sad facial expressions on the perception of time in Parkinson's disease patients with mild cognitive impairment. Journal of Clinical and Experimental Neuropsychology, 22, 1-16.

Perbal, S., J. Couillet, P. Azouvi, \& V. Pouthas (2003). Relationship between time estimation, memory, attention, and processing speed in patients with severe traumatic brain injury. Neuropsychologia 41, 1599-1610.

Perbal, S., B. Deweer, B. Pillon, M. Vidailhet, B. Dubois, \& V. Pouthas (2005). Effects of internal clock and memory disorders on duration reproductions and duration productions in patients with Parkinson's disease. Brain \& Cognition, 58(1), 35-48.

Piras, F., F. Piras, V. Ciullo, E. Danese, C. Caltagirone, \& G. Spalletta (2014). Time dysperception perspective for acquired brain injury. Frontiers in Neurology, 4, 217.

Rammsayer, T.H. (2008). Neuropharmacological approaches to human timing. In Grondin, S. (Ed.), Psychology of Time (pp. 295-320). Bingley: Emerald Group.

Rattat, A.C. \& S. Droit-Volet (2012). What is the best and easiest method of preventing counting in different temporal tasks? Behavioural Research Methods, 44(1), 67-80.

Schmitter-Edgecombe, M., \& A.D. Rueda (2008). Time estimation and episodic memory following traumatic brain injury. Journal of Clinical Experimental Neuropsychology, 30, 212-223.

Stuss, D.T., L. Stethem, H. Hugenholtz, T. Picton, J. Pivik, \& M.T. Richard (1989). Reaction time after head injury: Fatigue, divided and focused attention, and consistency of performance. Journal of Neurology, Neurosurgery, and Psychiatry, 52, 742-748.

Thoenes, S., \& D. Oberfeld (2017). Meta-analysis of time perception and temporal processing in schizophrenia: Differential effects on precision and accuracy. Clinical Psychology Review, 54, 44-64.

Thönes, S., \& D. Oberfeld (2015). Time perception in depression: A meta-analysis. Journal of Affective Disorders, 175, 359-372.

Toplak, M.E., C. Dockstader, \& R. Tannock (2006). Temporal information processing in ADHD: Findings to-date and new methods. Journal of Neuropsychological Methods, $15,15^{-29 .}$

Triesman, M. (1963). Temporal discrimination and the indifference interval. Implication for model of the "internal clock". Psychological Monographs, 77, 1-31.

Ulrich, R., J. Nitschke, \& T. Rammsayer (2006). Crossmodal temporal discrimination: Assessing the predictions of a general pacemaker-counter model. Perception \& Psychophysics, 68, 1140-1152. 
Zakay, D. (1990). The evasive art of subjective time measurement: Some methodological dilemmas. In Block, R.A. (Ed.), Cognitive models of psychological time (pp. 59-84). Lawrence Erlbaum Associates.

Zakay, D. (1993). Time estimation methods: Do they influence prospective duration estimates? Perception, 22, 91-101.

Zakay, D., \& R.A. Block (1996). The role of attention in time estimation processes. In Pastor, M.A. \& J. Artieda (Eds.), Time, internal clocks and movement (pp. 143-163). Amsterdam: Elsevier.

Zakay, D., \& R.A. Block (2004). Prospective and retrospective duration judgments: An executive-control perspective. Acta Neurobiologiae Experimentalis, 64, 319-328. 